\title{
Ensayos de hormigones fabricados con polvo de corcho
}

\section{Tests on concrete containing cork powder admixtures}

\author{
B. González(*), B. Llamas(**), A. Juan ${ }^{(*)}$ e I. Guerra(*)
}

Recepción/Received: 11-III-05

Aceptación/Accepted: 20-X-06

RESUMEN

Este trabajo de investigación tiene por objeto conocer algunas propiedades físicas y mecánicas de un hormigón elaborado en laboratorio, adicionándole diversas proporciones de polvo de corcho. Las propiedades del material resultante, si bien carecen de la resistencia mecánica que caracteriza al hormigón, parecen interesantes para su uso en ciertas aplicaciones de la ingeniería agronómica tales como en la fabricación de piezas para solados de parques infantiles y jardines, o en los cubículos de ciertas construcciones ganaderas, extremos que es preciso analizar y comprobar.

Palabras clave: hormigón, material orgánico, tratamiento de residuos, propiedades mecánicas, caracterización.

\section{SUMMARY}

The present study aimed to determine the physical and mechanical properties of laboratory concrete made with different proportions of cork powder. While the resulting material lacked the mechanical strength characteristic of concrete, its properties may prove to be apt for certain hardscaping and agricultural uses, such as in the manufacture of pavement for playgrounds and parks, or certain kinds of structures used in livestock raising. These findings need to be analyzed and verified.

Keywords: concrete, organic material, waste treatment, mechanical properties, characterization.

\section{INTRODUCCIÓN}

Es habitual en nuestra sociedad industrial hacer un estudio previo de las repercusiones que un nuevo proyecto de ingeniería puede tener sobre el medio. De hecho, la legislación española sobre medio ambiente obliga a hacer, en muchos casos, un estudio de evaluación de impacto ambiental para conocer la repercusión que tanto las obras como la propia actividad industrial tienen en el medio. Dentro de esta creciente sensibilización social por los temas medioambientales, la reutilización y el reciclado de residuos agrícolas e industriales se ofrecen como una de las mejores alternativas para reducir la contaminación ambiental.

La acumulación de algunos materiales de desecho ha propiciado que se busquen nuevas aplicaciones en el campo de la construcción. Así, cabe citar algunos trabajos de investigación encaminados a encontrar la forma de reciclar hormigones procedentes de demoliciones como sustitutos de áridos en la dosificación de nuevos hormigones (1), o la utilización de lodos de depuradoras como adiciones en la fabricación de adoquines prefabricados de hormigón (2).

(*) Escuela Superior y Técnica de Ingeniería Agraria, León (España).

(**) Instituto Energético y Minero (ENERMITEC), León (España). 
En este trabajo nos proponemos evaluar las propiedades del hormigón compuesto por pequeñas adiciones de polvo de corcho procedente de la industria de la madera. El aspecto de las primeras plaquetas elaboradas en el laboratorio, tanto por su cálida textura, como por su aparente resistencia, hicieron pensar en la posibilidad de hallar alguna utilidad, de tal manera que el producto resultante reuniera la doble condición de ser un producto ecológico, pues reutiliza un residuo industrial y funcional, al ser capaz de prestar un servicio.

Desde hace tiempo, en el campo de la Ingeniería Agronómica, se vienen diseñando los suelos de las granjas de hormigón en masa, obviando algunos problemas que presenta para ciertas especies ganaderas. El hormigón en masa es un material resistente, duradero, fácil de limpiar y cuya ejecución no presenta problemas de ningún tipo. Sin embargo, resulta ser demasiado duro y resbaladizo para las pezuñas del ganado vacuno lechero, lo que a veces ocasiona problemas de cojera. Vermunt y Greenough (3) consideran que la cojera en las vacas lecheras es un serio problema de higiene animal en muchas granjas europeas, y esto supone cuantiosas pérdidas económicas para los ganaderos. Nele de Belie (4) estudió el comportamiento biomecánico de las vacas y el efecto producido en las pezuñas en función del tipo de suelo. Concluye que el acabado superficial del suelo tiene gran importancia en la presión soportada en la parte de la pezuña en contacto con el suelo. Un material más blando y rugoso que el hormigón podría paliar el daño de las pezuñas de los bovinos. La adición de polvo de corcho al hormigón permitiría unos acabados superficiales menos lesivos para estos animales. Lógicamente, siempre que el material resultante mantenga unas condiciones adecuadas de resistencia mecánica y durabilidad. Cabe pensar, conociendo las propiedades del corcho, que eso es posible.

El corcho es un tejido vegetal constituido por la agrupación de células muertas (40 millones $/ \mathrm{cm}^{3}$ ), con forma, estructura y disposición muy particular, lo que le confiere unas características muy interesantes para la elaboración de algunos materiales de construcción. Cabe destacar su gran elasticidad, su capacidad de aislamiento térmico y acústico, su durabilidad y, por último, su origen natural: procede del alcornoque (Quercus suber L.). Es un material de baja densidad, entre 0,10 y $0,20 \mathrm{~kg} / \mathrm{dm}^{3}$, capaz de soportar presiones considerables, superiores a $90 \mathrm{MPa}, \mathrm{y}$ con una gran capacidad de recuperación de su forma original una vez que cesan las presiones.

Siguiendo las advertencias de Sousa (5) sobre la conveniencia de hacer los ensayos adecuados a los hormigones que utilizan adiciones procedentes de materiales reciclados, en este artículo se muestran los resultados obtenidos de los ensayos de varias dosificaciones de hormigón con cantidades variables de polvo de corcho. Los objetivos que nos hemos planteados en este trabajo son: conocer cómo influyen diversas proporciones de polvo de corcho en las propiedades del hormigón resultante; establecer un procedimiento para elaborar (amasar) adecuadamente los componentes de este hormigón; y, en tercer lugar, caracterizar este material para poder determinar o descartar algunas aplicaciones constructivas.

\section{PARTE EXPERIMENTAL}

\subsection{Materiales}

En este trabajo se evaluaron seis grupos de hormigones que tienen en su composición diferentes cantidades de polvo de corcho (véase la Tabla 2). El corcho no es un sustituto del árido, sino que ha sido añadido como un componente adicional a la dosificación preestablecida de un hormigón base o de referencia.

La dosificación del hormigón de referencia fue realizada a partir del método del módulo granulométrico de De la Peña, según se describe en la referencia (6): la composición cuantitativa del hormigón se muestra en la Tabla 2. Se empleó un cemento CEM I 52.5R de peso específico $3,1 \mathrm{~kg} / \mathrm{L}$. Se fijó una resistencia característica de $20 \mathrm{~N} / \mathrm{mm}^{2}$, ya que la utilidad que se pretende dar a este tipo de hormigones no es estructural y, por tanto, no es necesaria una resistencia mayor. Se buscó una consistencia plástica, con el fin de facilitar la puesta en obra. Sin embargo,

Tabla 1. Curva granulométrica del polvo de corcho

\begin{tabular}{|c|c|c|c|c|c|c|c|c|}
\hline \multirow[t]{2}{*}{ Luz (mm) } & \multirow[t]{2}{*}{ Tamaño máx. } & \multirow[t]{2}{*}{ Muestra $(g)$} & \multicolumn{2}{|c|}{ Retenido } & \multicolumn{2}{|c|}{$\%$ Retenido } & \multicolumn{2}{|c|}{$\%$ Pasa } \\
\hline & & & Parcial & Acumulado & Parcial & Acumulado & Parcial & Acumulado \\
\hline 0,63 & \multirow{7}{*}{0,125} & \multirow{7}{*}{100} & 0,02 & 0,02 & 0,02 & 0,018 & 99,982 & 100,0 \\
\hline 0,32 & & & 0,10 & 0,12 & 0,10 & 0,118 & 99,900 & 99,9 \\
\hline 0,125 & & & 14,30 & 14,42 & 14,30 & 14,418 & 85,700 & 85,6 \\
\hline 0,08 & & & 14,90 & 29,32 & 14,90 & 29,318 & 85,100 & 70,7 \\
\hline 0,063 & & & 14,50 & 43,82 & 14,50 & 43,818 & 85,500 & 56,2 \\
\hline 0,04 & & & 28,30 & 72,12 & 28,30 & 72,118 & 71,700 & 27,9 \\
\hline colector & & & 26,40 & 98,52 & 26,40 & 98,518 & 73,600 & 1,5 \\
\hline
\end{tabular}


tal como muestran los resultados de la Tabla 3, fue imposible lograr este tipo de consistencia.

Se emplearon áridos rodados de naturaleza silícea, de tamaño máximo $20 \mathrm{~mm}$. En las Figuras 1 y 2, se muestran las curvas granulométricas de la arena y de la grava. Se determinó el peso específico del árido fino, 2,52 $\mathrm{kg} / \mathrm{L}$, y el del árido grueso, 2,56 kg/L. También se determinó la humedad de la arena, 1,32\%, y de la grava, $0,23 \%$, con el fin de hacer las correcciones oportunas al agua de amasado. Todo ello se llevó a cabo siguiendo las normas UNE 83-133-90 y UNE 83-134-90 (7 y 8).

Se determinó el coeficiente de Los Ángeles de la grava y la friabilidad de la arena siguiendo lo establecido en las normas UNE 83-116-90 (9) en el primer caso y la UNE 83-11589 en el segundo (10). La grava tiene un coeficiente de Los Ángeles de 42, y el coeficiente de friabilidad de la arena ha sido de 11. Ambos cumplen lo establecido por la EHE.

La relación agua/cemento de la mezcla en el hormigón de referencia fue de 0,6. Al llevar a cabo en el laboratorio el amasado del hormigón con corcho, surgieron ciertos problemas debidos a la gran capacidad que tiene el polvo de corcho de absorber agua. Se sabe que el corcho es un material muy estable y, por tanto, no se preveían problemas en cuanto a la posibilidad de que pudiera reaccionar químicamente con el cemento durante la fase de fraguado y endurecimiento; y así fue. Sin embargo, lo que no podíamos imaginar es la gran avidez del polvo de corcho por el agua. Los primeros intentos de dosificación fallaron por completo: el polvo de corcho absorbía la mayor parte del agua de la dosificación, impidiendo el amasado.

\subsection{Métodos}

En este trabajo, desde el principio, se había descartado el uso de cualquier tipo de aditivos, pero llegado al punto en el que resultaba imposible lograr un buen amasado de la mezcla, el equipo de investigación se planteó seriamente utilizar fluidificantes. A pesar de estos problemas iniciales, el trabajo continuó según lo previsto: se prescindió de los aditivos, pero hubo que hacer correcciones en la cantidad de agua y modificaciones en el protocolo del amasado.

En cuanto a las correcciones de la dosificación de agua establecida para el hormigón base, se optó por incrementar su dosificación en el hormigón aligerado con el polvo de corcho, pues de otro modo no parecía posible lograr un

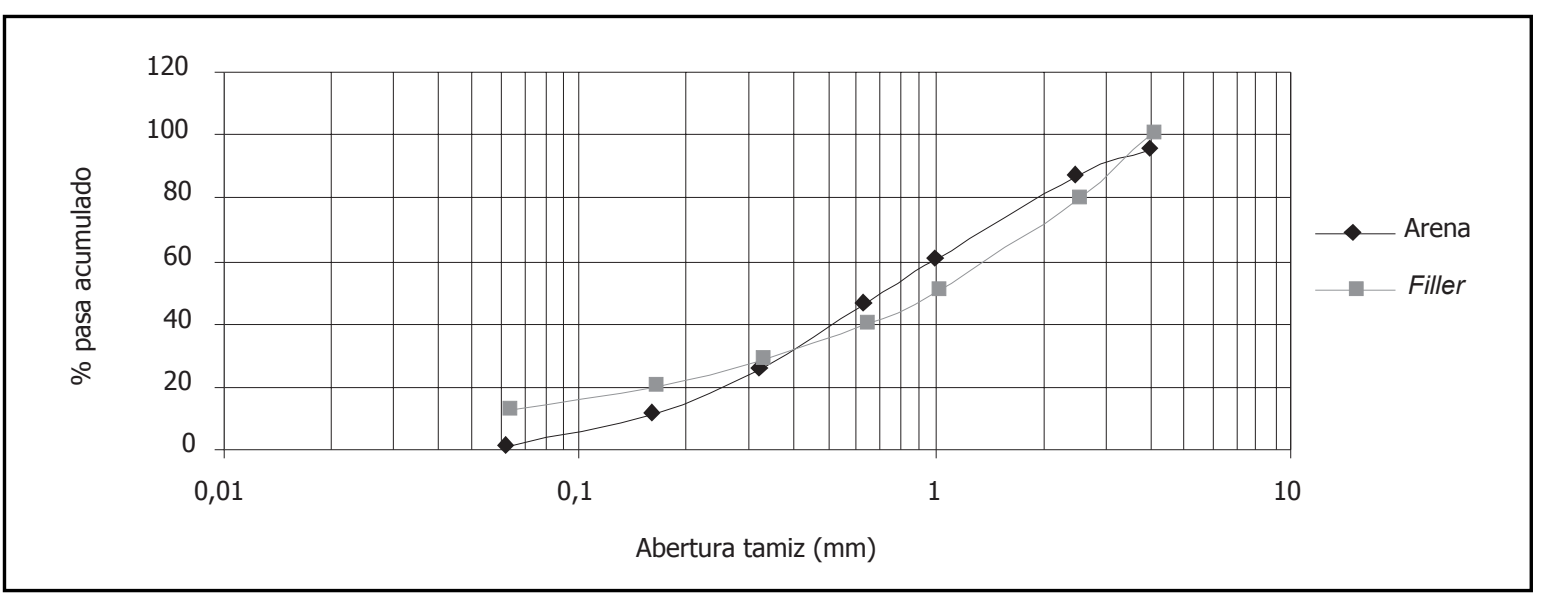

Figura 1. Curva granulométrica de la arena.

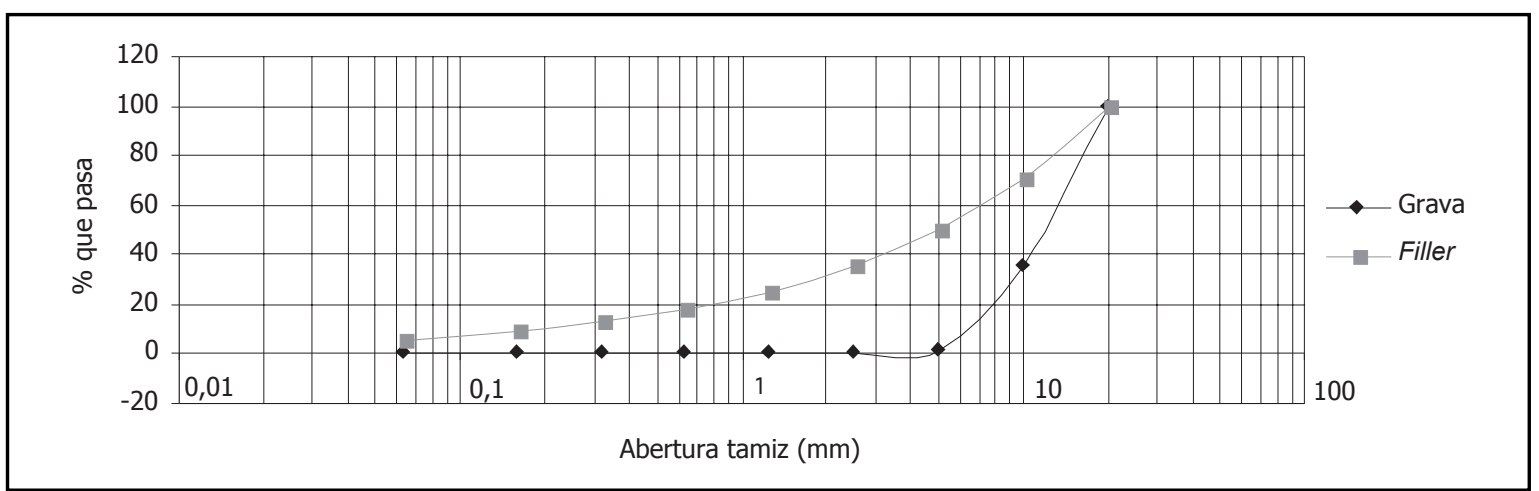

Figura 2. Curva granulométrica de la grava. 
Tabla 2

Componentes del hormigón base

\begin{tabular}{|l|c|c|c|}
\hline Componentes & Densidad $\mathbf{( g / \mathbf { c m } ^ { 3 } )}$ & $\mathbf{( \mathbf { L } / \mathbf { m } ^ { \mathbf { 3 } } )}$ & $\mathbf{( \mathbf { k g } / \mathbf { m } ^ { \mathbf { 3 } } )}$ \\
\hline Arena & 2,52 & 311 & 784 \\
\hline Grava & 2,56 & 434 & 1.111 \\
\hline Cemento & 3,10 & 95 & 295 \\
\hline Agua & & 185 & 185 \\
\hline
\end{tabular}

(*) Se utilizó un tipo de cemento sin adiciones: CEM-I.

buen amasado. Tras varias pruebas en el laboratorio, se decidió incrementar el volumen de agua una cantidad equivalente al $250 \%$ del peso de corcho. En la Tabla 3 se muestra la cantidad de polvo de corcho y la cantidad adicional de agua que se añadió a la dosificación preestablecida en la Tabla 2, para el hormigón de referencia.

En cuanto al protocolo seguido para el amasado, después de varias pruebas, no se encontró otra forma práctica de hacerlo, que mezclando primeramente en seco el árido con el polvo de corcho, siguiendo el siguiente protocolo:

1. Mezclado del árido fino, del árido grueso y del polvo de corcho durante 10 minutos.

2. Humidificación de las paredes, de las aspas y del fondo de hormigonera.

3. Introducción del cemento y de la mitad del agua de dosificación en la hormigonera.

4. Mezclado hasta conseguir una masa homogénea. Se emplearon otros 10 minutos.

5. Adición de la mezcla de áridos y polvo de corcho, junto con el agua restante.

6. Amasado durante 10 minutos.

7. Medición de la consistencia de la masa mediante el cono de Abrams.

8. Posteriormente, se procedió a la toma de muestras del hormigón fresco (11), y al llenado de los moldes de las probetas (12). Dado que el asiento en cono fue muy pequeño en las muestras con polvo de corcho, se empleó un vibrador de aguja.

De esta forma se consiguió una masa homogénea.

Los áridos fueron cuarteados y posteriormente tamizados con tamices de la serie UNE: se hizo siguiendo las normas

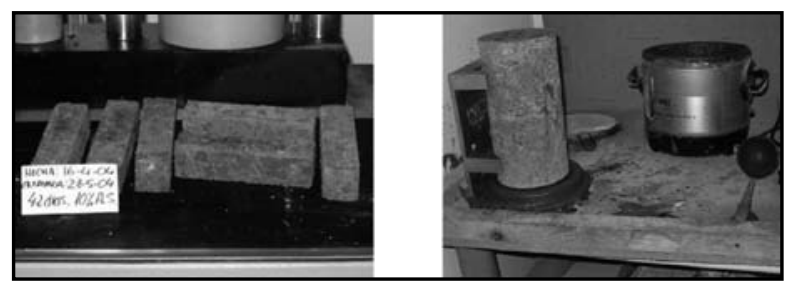

Figura 3. Probetas prismáticas y cilíndricas con material para el refrentado.
EN 933-2 (13) para al tamizado, y el programa prEN 932-2 (14) en el cuarteado. Los equipos empleados en estos ensayos de caracterización de los áridos fueron calibrados previamente, siguiendo el programa prEN 932-5 (15).

Tal como se indicó anteriormente, para cada tipo de dosificación se elaboraron 15 probetas cilíndricas (16) de 15 x 30 centímetros para los ensayos de compresión, según la UNE-83-304-84 (17), y de tracción indirecta UNE 83-306-85 (18). Las probetas se conservaron 48 horas a una temperatura de aproximadamente $20^{\circ} \mathrm{C}$; posteriormente, una vez desmoldadas, se trasladaron a una cámara de conservación con una humedad relativa de $95 \%$ y a una temperatura de $20^{\circ} \mathrm{C}$.

Con el fin de obtener más información sobre este material, el equipo de investigación se planteó la posibilidad de hacer algún otro tipo de ensayo, y se optó por llevar a cabo dos más: el ensayo de flexo-tracción y posterior compresión (19), y el ensayo de rotura por impacto (20). Es cierto que no son ensayos propios de hormigones sino de morteros, el primero, y de rocas, el segundo. Pero se optó definitivamente por hacerlos, ya que una de las posibles aplicaciones de este hormigón, con adiciones de corcho, puede estar en la fabricación de plaquetas: plaquetas para solados de granjas, parques y jardines, donde pueden ser habituales cargas concentradas que provoquen esfuerzos de flexión y de impacto. Este comportamiento puede ser caracterizado de forma más aproximada mediante estos dos ensayos que con el de compresión simple.

La elaboración de las probetas prismáticas se llevó a cabo de la siguiente manera: preparada la masa, y comprobado que el molde y la tolva están firmemente unidos a la mesa compactadora, se vierte la masa y se compacta; para ello, un motor eléctrico acciona la leva de la compactadora a una velocidad uniforme de una revolución por segundo. Un dispositivo de control y un contador aseguran que el periodo de compactación sea exactamente de sesenta golpes. Posteriormente, se retira el molde de la mesa de sacudidas, se separa la tolva y se quita el exceso de masa, enrasando con una regla plana

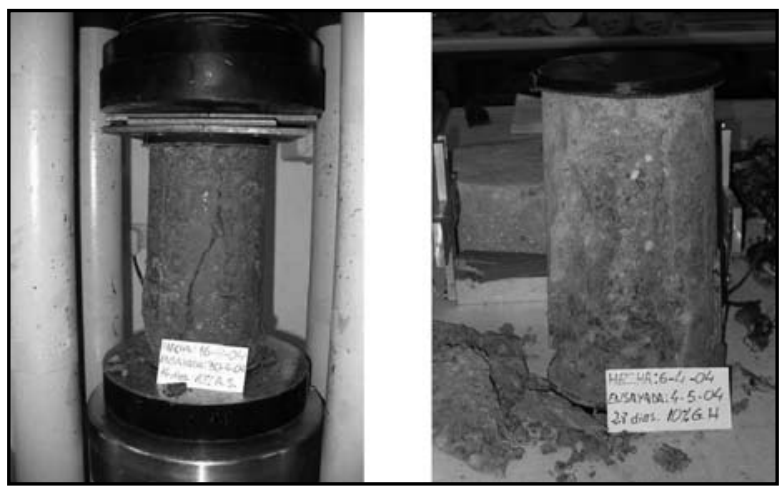

Figura 4. Prensa de ensayo a compresión y probetas ensayadas. 
Tabla 3. Adiciones de corcho e incremento de la dosis de agua

\begin{tabular}{|l|c|c|c|c|}
\hline Componentes & $\mathbf{( k g / \mathbf { m } ^ { 3 } )}$ & $\mathbf{( L / \mathbf { m } ^ { \mathbf { 3 } } )}$ & Incremento de la dosis de agua $\left.\mathbf{(}^{*}\right)$ & $\mathbf{A} / \mathbf{C}$ \\
\hline $10 \%$ de corcho sobre grava & 111 & 555 & 277 & 1,57 \\
\hline $10 \%$ de corcho sobre áridos & 190 & 947 & 475 & 2,24 \\
\hline $5 \%$ de corcho sobre áridos & 297 & 96 & 237 & 1,42 \\
\hline $8 \%$ de corcho sobre áridos & 151 & 756 & 380 & 1,91 \\
\hline
\end{tabular}

${ }^{*}$ ) Es un $250 \%$ respecto a la cantidad de corcho en peso.

Tabla 4. Dimensiones de las probetas en el ensayo de impacto

\begin{tabular}{|l|c|c|c|c|}
\hline Probetas & $\mathbf{N}^{\mathbf{0}}$ de probetas por cada tipo de hormigón & Base inferior (mm) & Base superior (mm) & Espesor (mm) \\
\hline Pequeñas & 2 & $193 \times 193$ & $170 \times 170$ & $47 \pm 2$ \\
\hline Grandes & 2 & $190 \times 390$ & $180 \times 370$ & $42 \pm 2$ \\
\hline
\end{tabular}

para conseguir una superficie lisa. Estas probetas se tapan y conservan hasta el desmoldeo, de forma similar a lo descrito más arriba para las probetas cilíndricas. La resistencia a flexión (expresada en forma de tensión) ha sido calculada mediante la fórmula siguiente [1]:

$$
R_{f}=\frac{1,5 F_{f} L}{b^{3}} \quad\left(N / m^{2}\right)
$$

Donde:

$b$ es el lado de la sección cuadrada del prisma en milímetros (mm).

$F_{f}$ es la carga de rotura en Newtons (N) aplicada en el plano mediatriz entre apoyos.

$L$ es la distancia entre soportes $(\mathrm{mm})$.

La resistencia compresión $\left(R_{c}\right)$ viene dada por la siguiente fórmula [2]:

$$
R_{c}=\frac{F_{c}}{1600} \quad\left(\mathrm{~N} / \mathrm{mm}^{2}\right)
$$

Donde:

$F_{C}$ es la carga de rotura $(\mathrm{N})$.

$1600=40 \mathrm{~mm} \times 40 \mathrm{~mm}$ es la superficie de los platos o placas auxiliares $\left(\mathrm{mm}^{2}\right)$.

En cuanto al ensayo de rotura por impacto, se llevó a cabo siguiendo la norma inglesa CEN prEN 00246-019 (17), aunque con ciertas modificaciones que se describen a continuación. Se emplea una bola esférica de metal de masa $1000 \pm 10 \mathrm{~g}$; un recipiente de dimensiones mínimas $40 \times 40 \times 15 \mathrm{~cm}$, en el cual se coloca una cama de 2 centímetros de arena de tamiz comprendido entre 2 y 0,076 mm; y por último, una guía-columna de 1,5 metros de altura y graduada cada $5 \mathrm{~cm}$. Para cada tipo de dosificación, incluido el hormigón de referencia, se fabricaron cuatro probetas tronco-piramidales: dos de tamaño grande y dos de tamaño pequeño, cuyas dimensiones se indican en la Tabla 4.
El procedimiento seguido durante este ensayo ha sido el siguiente: se coloca la pieza a ensayar, la plaqueta, en la cama de arena y se nivela hasta que quede perfectamente horizontal. Se deja caer la bola de acero sobre dicha pieza desde una altura inicial de $10 \mathrm{~cm}$. Si la pieza no rompe, se va incrementando la altura de caída de 5 en $5 \mathrm{~cm}$ hasta que se produzca la rotura de la pieza. Se procura que todos los impactos tengan lugar en la región central de las placas, y nunca a menos de 3 centímetros de cualquiera de sus bordes. El lugar de cada impacto en la plaqueta debe distar al menos $5 \mathrm{~cm}$ del anterior. El ensayo con la segunda plaqueta comienza a una altura de $15 \mathrm{~cm}$ por debajo de la altura de rotura de la primera, y si no fuera posible, de $10 \mathrm{~cm}$. Si alguna pieza rompe en el primer impacto, el resultado será rechazado. El cálculo de la energía se hace a partir de la conocida fórmula de la energía potencial: $E(J)=m g h$.

\section{RESULTADOS Y DISCUSIÓN}

En la Tabla 5 se muestra la consistencia medida con el cono de Abrams. La gran avidez del corcho por el agua hace que todas las muestras, excepto la del hormigón de referencia, sean de consistencia seca. En general, se puede afirmar que estos hormigones con polvo de corcho van a tener en común una consistencia seca, lo que en estas condiciones va a dificultar su trabajabilidad.

Los resultados obtenidos en los ensayos de compresión figuran en la Tabla 6. En esta tabla se muestra el coeficiente de variación correspondiente a las probetas rotas a 42 días. Las probetas de menor edad tuvieron resistencias demasiado bajas.

La resistencia final del hormigón resultante es muy baja: en el mejor de los casos se llega a los 4,3 MPa (casi un 20\% de la del hormigón de referencia). Estos resultados descartan cualquier posibilidad de utilizar este tipo de hormigones como material estructural. Como material no estructural, se puede aprovechar su reducida capacidad resistente a partir 
Tabla 5. Consistencia de las probetas de hormigón (C. de Abrams)

\begin{tabular}{|l|c|c|}
\hline Muestras & Asiento $\mathbf{( c m )}$ & Consistencia \\
\hline Hormigón de Referencia & 6,5 & Blanda \\
\hline $10 \%$ sobre grava en húmedo & 0,8 & Seca \\
\hline $10 \%$ sobre árido en húmedo & 0 & Seca \\
\hline $10 \%$ sobre grava en seco & 0,7 & Seca \\
\hline $10 \%$ sobre áridos en seco & 0,5 & Seca \\
\hline $5 \%$ sobre áridos en seco & 0,3 & Seca \\
\hline $8 \%$ sobre áridos en seco & 0 & Seca \\
\hline
\end{tabular}

de, como mínimo, 42 días de edad. El corcho, debido a su alta capacidad de retención de agua, influye notablemente en la ralentización del curado. Cabe pensar que es la alta relación a/c muy superior a 0,6 (véase la Tabla 3) la principal razón de esta disminución de la velocidad de curado. Los mejores resultados de la resistencia a compresión han sido obtenidos en las muestras fabricadas con un $10 \%$ de polvo de corcho (sobre el peso en seco de la grava): $\mathrm{f}_{\mathrm{cm}}=4,3 \mathrm{MPa}$; además, muestran ser los de menor dispersión.

A la vista de los resultados, es evidente que el aprovechamiento del corcho trae consigo la pérdida de resistencia del hormigón estructural. Este material, que se podría calificar de ecoeficiente, ya que aprovecha un residuo industrial, no es apto para fines estructurales. Sin embargo, cabe la posibilidad de ser utilizado en el mundo de la agroingeniería como material no estructural en solados de parques y jardines, camas de cubículos de ganado vacuno, etc., extremos estos que es preciso analizar y comprobar.

La tensión media $\mathrm{f}_{\mathrm{cm}}$ y el coeficiente de variación $\delta$ han sido calculados según lo establecido por la norma EHE [3 y 4]:

$$
\begin{gathered}
f_{c m}=\frac{f_{c 1}+f_{c 2}+\ldots+f_{c n}}{n} \\
\delta=\sqrt{\frac{1}{n} \sum_{i=1}^{n}\left(\frac{f_{c_{i}}-f_{c_{m}}}{f_{c_{m}}}\right)^{2}}
\end{gathered}
$$

Tabla 7. Resultados del ensayo brasileño

\begin{tabular}{|l|c|c|}
\hline $\begin{array}{l}\text { Muestras con 42 días de edad } \\
\text { (Excepto hormigón de } \\
\text { referencia de 28 días) }\end{array}$ & Carga (kN) & Tensión (MPa) \\
\hline Hormigón de Referencia & 150,1 & 2,12 \\
\hline $10 \%$ sobre grava en húmedo & 27,3 & 0,39 \\
& 26,8 & 0,38 \\
\hline $10 \%$ sobre árido en húmedo & 0,0 & \\
\hline $10 \%$ sobre grava en seco & 0,0 & 53,3 \\
\hline $10 \%$ sobre áridos en seco & 54,7 & 0,75 \\
& 21,6 & 0,77 \\
\hline $5 \%$ sobre áridos en seco & 19,5 & 0,28 \\
\hline $8 \%$ sobre áridos en seco & 39,4 & 0,56 \\
& 44,1 & 0,62 \\
\hline
\end{tabular}

$(*)$ Una de las probetas ( $10 \%$ sobre árido en húmedo) se rompió antes de comenzar el ensayo.

En la Tabla 6 se puede observar que la uniformidad de las probetas dosificadas con un $10 \%$ de corcho sobre grava en seco, además de ser las que tienen las mayores tensiones de rotura a compresión a 42 días, muestran una excelente uniformidad (0,02\%). Según la EHE la uniformidad es excelente cuando $\delta(\%)$ es inferior al $10 \%$.

Los resultados obtenidos en el ensayo brasileño, realizado a 28 días de edad en el caso del hormigón de referencia y a 42 días en el de hormigón con polvo de corcho, se muestran en la Tabla 7. Como puede verse, el polvo de corcho supone una notable reducción de la ya de por sí escasísima resistencia a la tracción indirecta que tiene el hormigón.

En el ensayo de flexo-tracción y posterior compresión, se utilizaron 18 probetas normalizadas, de 42 días de edad; los resultados figuran en la Tabla 8.

Los resultados del ensayo de impacto se muestran en la Tabla 9. Como puede verse, existe una cierta correlación

\begin{tabular}{|c|c|c|c|c|}
\hline$f_{c m}$ : Tensión media & 14 días & 28 días & \multicolumn{2}{|c|}{42 días } \\
\hline Muestras & $\mathbf{f}_{\mathrm{cm}} \mathrm{MPa}$ & $\mathbf{f}_{\mathrm{cm}} \mathrm{MPa}$ & $\mathbf{f}_{\mathrm{cm}} \mathrm{MPa}$ & Coef. de variación $(\delta)$ \\
\hline Número de probeta & 2 & 3 & $5(*)$ & \\
\hline Hormigón de Referencia & 18,3 & 23,2 & & \\
\hline $10 \%$ sobre grava en húmedo & 6,8 & 6,6 & 2,03 & 0,10 \\
\hline $10 \%$ sobre árido en húmedo & 0,0 & 0,0 & 0,80 & 0,06 \\
\hline $10 \%$ sobre grava en seco & 3,7 & 3,6 & 4,30 & 0,02 \\
\hline $10 \%$ sobre áridos en seco & 1,5 & 0,8 & 1,57 & 0,18 \\
\hline $5 \%$ sobre áridos en seco & 5,0 & 5,3 & 3,80 & 0,21 \\
\hline $8 \%$ sobre áridos en seco & 0,0 & 1,0 & 2,00 & 0,39 \\
\hline
\end{tabular}

Tabla 6. Resultados del ensayo de compresión

$\left(^{*}\right)$ Excepto el hormigón de referencia. 
con el ensayo de compresión. Las muestras con un 5\% de corcho sobre árido seco y las del $10 \%$ sobre grava en seco dan los mejores resultados a compresión.

La energía de rotura de las placas o plaquetas varía en función de la dosificación de las muestras, tal como muestra la Tabla 9. Existe una gran variabilidad entre los resultados de los distintos tipos de dosificación. Los valores más altos vuelven a ser los de las muestras con una cantidad de polvo de corcho de un $5 \%$ respecto al árido en seco, y de un $10 \%$ respecto de la grava en seco. Como cabía esperar, las placas pequeñas tienen mejor comportamiento ante el impacto que las grandes.

Estos dos hormigones, y sobre todo el que tiene un 5\% de corcho, son tal vez los que han mostrado los mejores resultados en estos ensayos preliminares. Este hormigón debería ser la base para posteriores ensayos, como durabilidad,

Tabla 8. Resultados del ensayo de flexo-tracción y compresión

\begin{tabular}{|l|c|c|c|c|}
\hline \multirow{2}{*}{ Muestras con 42 días de edad } & \multicolumn{2}{|c|}{ Ensayo de Flexión } & \multicolumn{2}{c|}{ Ensayo de Compresión } \\
\cline { 2 - 5 } & Carga $(\mathbf{k N})$ & Tensión $\mathbf{( M P a )}$ & Tensión (MPa) & Coeficiente de variación $(\delta$ ) \\
\hline $10 \%$ sobre grava en húmedo & 0,775 & 1,82 & 5,32 & 0,17 \\
\hline $10 \%$ sobre árido en húmedo & 0,20 & 0,46 & 1,53 & 0,02 \\
\hline $10 \%$ sobre grava en seco & 0,83 & 1,94 & 6,33 & 0,98 \\
\hline $10 \%$ sobre áridos en seco & 0,29 & 0,68 & 1,78 & 0,02 \\
\hline $5 \%$ sobre áridos en seco & 0,80 & 1,87 & 5,78 & 0,14 \\
\hline $8 \%$ sobre áridos en seco & 0,40 & 0,93 & 2,68 & 0,11 \\
\hline
\end{tabular}

Nota: Cada muestra consta de 3 probetas prismáticas de $4 \times 4 \times 16 \mathrm{~cm}$ rotas a flexión y después a compresión.

Tabla 9. Ensayo de impacto con una bola de $1 \mathrm{~kg}$ de masa

\begin{tabular}{|c|c|c|c|c|c|}
\hline \multirow[t]{2}{*}{ Muestras } & \multirow[t]{2}{*}{ Tipo } & \multicolumn{2}{|c|}{ Altura caída de la bola } & \multirow[t]{2}{*}{ E. cinética (J) } & \multirow[t]{2}{*}{ Promedio (J) } \\
\hline & & Inicial (m) & Final (m) & & \\
\hline \multirow{4}{*}{ Hormigón de Referencia } & P & 0,10 & 0,90 & 8,82 & 9,80 \\
\hline & P & 0,75 & 1,10 & 10,78 & \\
\hline & G & 0,10 & 0,70 & 6,86 & 7,84 \\
\hline & G & 0,55 & 0,90 & 8,82 & \\
\hline \multirow{4}{*}{$10 \%$ sobre grava en húmedo } & $\mathrm{P}$ & 0,10 & 0,65 & 6,37 & 5,88 \\
\hline & $P$ & 0,50 & 0,55 & 5,39 & \\
\hline & G & 0,10 & 0,55 & 5,39 & 5,15 \\
\hline & G & 0,40 & 0,50 & 4,9 & \\
\hline \multirow{4}{*}{$10 \%$ sobre árido en húmedo } & $P$ & 0,10 & 0,25 & 2,45 & \\
\hline & $\mathrm{P}$ & no hecha & & & \\
\hline & G & 0,10 & 0,15 & 1,47 & 1,23 \\
\hline & G & 0,10 & 0,10 & 0,98 & \\
\hline \multirow{4}{*}{$10 \%$ sobre grava en seco } & $P$ & 0,10 & 0,70 & 6,86 & 6,62 \\
\hline & $P$ & 0,55 & 0,65 & 6,37 & \\
\hline & G & 0,30 & 0,45 & 4,41 & 3,92 \\
\hline & $\mathrm{G}$ & 0,30 & 0,35 & 3,43 & \\
\hline \multirow{4}{*}{$10 \%$ sobre áridos en seco } & $P$ & 0,10 & 0,40 & 3,92 & 4,41 \\
\hline & $P$ & 0,25 & 0,50 & 4,90 & \\
\hline & G & 0,10 & 0,15 & 1,47 & 1,96 \\
\hline & G & 0,10 & 0,25 & 2,45 & \\
\hline \multirow{4}{*}{$5 \%$ sobre áridos en seco } & $P$ & 0,10 & 0,75 & 7,35 & 7,35 \\
\hline & $\mathrm{P}$ & 0,60 & 0,75 & 7,35 & \\
\hline & G & 0,10 & 0,55 & 5,39 & 4,90 \\
\hline & G & 0,40 & 0,45 & 4,41 & \\
\hline \multirow{4}{*}{$8 \%$ sobre áridos en seco } & $\mathrm{P}$ & 0,10 & 0,45 & 4,41 & 4,41 \\
\hline & $P$ & 0,30 & 0,45 & 4,41 & \\
\hline & G & 0,10 & 0,50 & 4,90 & 4,66 \\
\hline & $\mathrm{G}$ & 0,30 & 0,45 & 4,41 & \\
\hline
\end{tabular}

P: probetas pequeñas. G: probetas grandes. Véase la tabla 6 . 

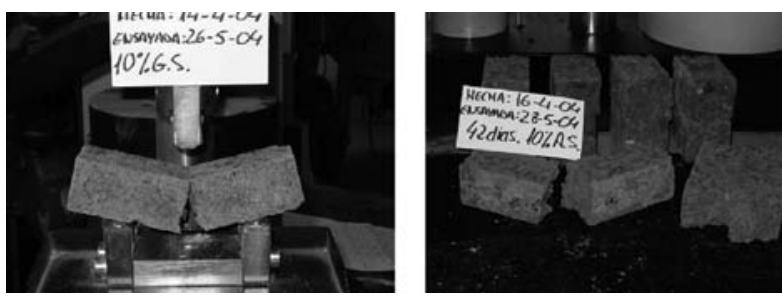

Figura 5. Ensayo a flexo-tracción y compresión.

desgaste, etc., y otros más específicos acordes con el uso definitivo que se vaya a dar a este material.

\section{CONCLUSIONES}

1. La resistencia final del hormigón resultante es muy baja: en el mejor de los casos, a 42 días, se llega a los 4,3 MPa. La presencia del corcho trae consigo la pérdida de resistencia que caracteriza al hormigón. Por lo tanto, no se puede utilizar nunca como hormigón estructural.
2. El hormigón con adiciones de polvo de corcho es de endurecimiento muy lento, debido a la alta cantidad de agua presente en la masa. Esto hace que haya que esperar, como mínimo, hasta los 42 días para romper las probetas, en lugar de a los 28 como establece la norma EHE.

3. La dosificación con polvo de corcho que ha proporcionado mejores resultados en estos ensayos preliminares, en general, es la que contiene un $10 \%$ de adiciones sobre grava en seco. Este hormigón, para usos no estructurales, debería ser la base para posteriores ensayos, como durabilidad, desgaste, etc., y otros más específicos acordes con el uso definitivo que se vaya a dar a este material.

4. Para conseguir un buen amasado, es necesario mezclar en seco el polvo de corcho con los áridos, añadiéndose lentamente a la masa agua-cemento e intercalando poco a poco el resto del agua. La dosificación de agua se debe incrementar un $250 \%$ para compensar la que es absorbida por el corcho.

\section{BIBLIOGRAFÍA / BIBLIOGRAPHY}

(1) Di Maio, A. A. y Traversa, L. P.: "Evaluación de hormigones reciclados mediante ensayos no destructivos", Mater. Construcc., vol. 53 (2003), no 271-272, pp. 37-46.

(2) Yagüe, A., Valls, S., Váquez, E. y Kuchinow, V.: "Utilización de lodo seco de depuradora de aguas residuales como adición en adoquines de hormigón prefabricado", Mater. Construcc., vol. 52 (2003), no 267, pp. 31-41.

(3)Vermunt, J. y Greenough, P. R.: "Claw conformation of dairy heifers in two management systems", British Veterinary Journal, no 152 (1996), pp. 321-331.

(4) De Belie, N. y Rombaut, E.: "Characterisation of Claw-floor contact pressures for standing cattle and the dependency on concrete roughness", Biosystems Engineering, no 85 (2003), pp. 339-346.

(5) Sousa, J. G. G., Bauer, E. y Sposto, M.: "Empleo de residuos en la construcción civil como áridos reciclados. Producción de bloques de hormigón", Mater. Construcc., vol. 53, no 271-272 (2003), pp. 59-70.

(6) Jiménez Montoya, P., García Meseguer, A. y Morán Cabré (2000): Cap. 3 "Dosificación del hormigón", en Hormigón armado, Editorial G.G., Barcelona.

(7) AENOR: UNE 83-133-90. Áridos para hormigones. Determinación de las densidades, coeficiente de agua en el árido fino, Madrid.

(8) AENOR: UNE 83-134-90. Áridos para hormigones. Determinación de las densidades, porosidad, coeficiente de absorción y contenido de árido grueso, Madrid.

(9) UNE 83- 116-90. Áridos para hormigones. Determinación del coeficiente "Los Ángeles", Madrid.

(10) AENOR: UNE 83-115- 89. Áridos para hormigones. Determinación del coeficiente de friabilidad de las arenas, Madrid.

(11) AENOR: UNE 83-300-84. Ensayos de hormigón. Toma de muestras de hormigón fresco, Madrid.

(12) AENOR: UNE 83.301-91/1R. Ensayos de hormigón. Fabricación y conservación de probetas, Madrid.

(13) AENOR: UNE-EN 933-2: 1997. Ensayos para determinación de las propiedades geométricas de los áridos. Parte 2: Determinación de la granulometría de las partículas. Tamices de ensayo, tamaño nominal de las aberturas, Madrid.

(14) AENOR: UNE prEN 932-2. Métodos de ensayo para determinar las propiedades generales de los áridos. Parte 2: Métodos para la reducción de muestras de laboratorio, Madrid.

(15) AENOR: UNE prEN 932-5. Métodos de ensayo para determinar las propiedades generales de los áridos. Parte 5: Equipo común y calibración, Madrid.

(16) AENOR: UNE EN 12390-1. Ensayos de hormigón endurecido. Forma, medida y otras características de las probetas y moldes, Madrid.

(17) AENOR: UNE 83-304-84 Ensayos de hormigón. Rotura por compresión, Madrid.

(18) AENOR: UNE 83-306-85. Ensayos de hormigón. Rotura por tracción indirecta (Ensayo Brasileño), Madrid.

(19) UNE-EN 196-1:1996. Métodos de ensayo de cementos. Parte 1: Determinación de resistencias mecánicas.

(20) CEN: PrEN 00246-019. Natural stones test methods. Energy of rupture by impact, Bruselas. 